\title{
ON $n$-IDEAL AMENABILITY OF CERTAIN BANACH ALGEBRAS
}

\author{
ZEINAB KAMALI ${ }^{\otimes}$ and MEHDI NEMATI
}

(Received 21 August 2011)

\begin{abstract}
In this paper we consider some notions of amenability such as ideal amenability, $n$-ideal amenability and approximate $n$-ideal amenability. The first two were introduced and studied by Gordji, Yazdanpanah and Memarbashi. We investigate some properties of certain Banach algebras in each of these classes. Results are also given for Segal algebras on locally compact groups.
\end{abstract}

2010 Mathematics subject classification: primary 46H05; secondary 43A07.

Keywords and phrases: Banach algebra, $n$-ideal amenability, abstract Segal algebra.

\section{Introduction}

Let $\mathcal{A}$ be a Banach algebra. Then the dual space $\mathcal{X}^{*}$ of a Banach $\mathcal{A}$-bimodule $\mathcal{X}$ is also a Banach $\mathcal{A}$-bimodule by the module actions

$$
\left\langle x^{*} \cdot a, x\right\rangle=\left\langle x^{*}, a \cdot x\right\rangle, \quad\left\langle a \cdot x^{*}, x\right\rangle=\left\langle x^{*}, x \cdot a\right\rangle
$$

for all $a \in \mathcal{A}, x \in \mathcal{X}, x^{*} \in \mathcal{X}^{*}$. In particular, for every $n \in \mathbb{N}$, the $n$th dual $\mathcal{X}^{(n)}$ of $\mathcal{X}$ is a Banach $\mathcal{A}$-bimodule and so, for every closed two-sided ideal $\mathcal{I}$ of $\mathcal{A}, \mathcal{I}$ is a Banach $\mathcal{A}$-bimodule and $\mathcal{I}^{(n)}$ is a dual Banach $\mathcal{A}$-bimodule for every $n \in \mathbb{N}$.

Let $\mathcal{X}$ be a Banach $\mathcal{A}$-bimodule. Then a continuous linear map $D: \mathcal{A} \rightarrow \mathcal{X}$ is called a derivation if

$$
D(a b)=a \cdot D(b)+D(a) \cdot b \quad(a, b \in \mathcal{A}) .
$$

For $x \in \mathcal{X}$, we define $\operatorname{ad}_{x}: \mathcal{A} \rightarrow \mathcal{X}$ as follows:

$$
\operatorname{ad}_{x}(a)=a \cdot x-x \cdot a \quad(a \in \mathcal{A}) .
$$

It is easy to show that $\operatorname{ad}_{x}$ is a derivation. Such derivations are called inner derivations.

A derivation $D: \mathcal{A} \rightarrow \mathcal{X}$ is called approximately inner if there exists a net $\left(x_{\alpha}\right) \subseteq \mathcal{X}$ such that

$$
D(a)=\lim _{\alpha}\left(a \cdot x_{\alpha}-x_{\alpha} \cdot a\right) \quad(a \in \mathcal{A}) .
$$

Recall that $\mathcal{A}$ is called amenable if each continuous derivation from $\mathcal{A}$ into each dual Banach $\mathcal{A}$-bimodule is inner. The algebra $\mathcal{A}$ is said to be weakly amenable if

(c) 2011 Australian Mathematical Publishing Association Inc. 0004-9727/2011 \$16.00 
each continuous derivation from $\mathcal{A}$ into the specific dual Banach $\mathcal{A}$-bimodule $\mathcal{A}^{*}$ is inner. The concept of weak amenability was first introduced by Bade et al. in [2] for commutative Banach algebras and was extended to the noncommutative case by Johnson in [11].

For a closed two-sided ideal $\mathcal{I}$ of $\mathcal{A}$, Gordji and Yazdanpanah [6] introduced and studied the notion of ideal amenability, $n$-ideal amenability and $n$ - $\mathcal{I}$-weak amenability of $\mathcal{A}$; see also [5]. In 2010, Mewomo [15] introduced the notion of approximate $\mathcal{I}$-weak amenability and approximate ideal amenability of Banach algebras.

Let $\mathcal{I}$ be a closed two-sided ideal of $\mathcal{A}$ and $n \in \mathbb{N}$. Then $\mathcal{A}$ is called $n$ - $\mathcal{I}$-weakly amenable if any continuous derivation from $\mathcal{A}$ into $\mathcal{I}^{(n)}$ is inner, $\mathcal{A}$ is called $n$-ideally amenable if $\mathcal{A}$ is $n$ - $\mathcal{I}$-weakly amenable for every closed two-sided ideal $\mathcal{I}$ of $\mathcal{A}$, and $\mathcal{A}$ is called permanently ideally amenable if it is $n$ - $\mathcal{I}$-weakly amenable for every closed two-sided ideal $\mathcal{I}$ of $\mathcal{A}$ and for each $n \in \mathbb{N}$.

Also, $\mathcal{A}$ is called approximately $n-\mathcal{I}$-weakly amenable if any continuous derivation from $\mathcal{A}$ into $\mathcal{I}^{(n)}$ is approximately inner. Then $n$-ideal amenability and approximate permanent ideal amenability of Banach algebras are defined similarly.

We briefly summarize the results of the paper. In Section 2 we give a sufficient condition for $n$-ideal amenability of the homomorphic image. We apply this result to obtain the $n$-ideal amenability of certain Banach algebras.

In Section 3 we investigate the relation between $n$-ideal amenability of $\mathcal{A}$ and approximate $n$-ideal amenability of abstract Segal algebras in $\mathcal{A}$. Then we apply these results to Segal algebras on a locally compact group.

\section{Permanent ideal amenability}

Let $\mathcal{A}$ and $\mathcal{B}$ be Banach algebras and let $\tau: \mathcal{A} \rightarrow \mathcal{B}$ be a bounded homomorphism. If $\mathcal{J}$ is a closed two-sided ideal of $\mathcal{B}$, then $\mathcal{J}^{(n)}$ is a Banach $\mathcal{A}$-bimodule by the module actions

$$
a \cdot F=\tau(a) \cdot F, \quad F \cdot a=F \cdot \tau(a) \quad\left(a \in \mathcal{A}, F \in \mathcal{J}^{(n)}\right) .
$$

It is easy to check that for each $n \geq 1, \tau^{(2 n)}: \mathcal{A}^{(2 n)} \rightarrow \mathcal{B}^{(2 n)}$, the $2 n$th dual operator of $\tau$, and $\tau^{(2 n-1)}: \mathcal{B}^{(2 n-1)} \rightarrow \mathcal{A}^{(2 n-1)}$, the $(2 n-1)$ th dual operator of $\tau$, are $\mathcal{A}$-bimodule morphisms.

Proposition 2.1. Let $\mathcal{A}$ and $\mathcal{B}$ be Banach algebras and let $\tau: \mathcal{A} \rightarrow \mathcal{B}$ and $\Phi: \mathcal{B} \rightarrow \mathcal{A}$ be bounded homomorphisms such that $\tau \circ \Phi=I_{\mathcal{B}}$. If $\mathcal{I}, \mathcal{J}$ are closed two-sided ideals of $\mathcal{A}$ and $\mathcal{B}$, respectively, such that $\tau(\mathcal{I}) \subseteq \mathcal{J}$ and $\Phi(\mathcal{J}) \subseteq \mathcal{I}$, then for each $n \in \mathbb{N}, \mathcal{B}$ is $n$ - $\mathcal{J}$-weakly amenable if $\mathcal{A}$ is $n$ - $\mathcal{I}$-weakly amenable.

Proof. Let $n \in \mathbb{N}$ and let $D: \mathcal{B} \rightarrow \mathcal{J}^{(n)}$ be a continuous derivation. Define $\widetilde{D}: \mathcal{A} \rightarrow$ $\mathcal{I}^{(n)}$ by

$$
\widetilde{D}(a)= \begin{cases}\left(\left(\left.\tau\right|_{\mathcal{I}}\right)^{(n)} \circ D \circ \tau\right)(a) & \text { if } n \text { is odd, } \\ \left(\left(\left.\Phi\right|_{\mathcal{J}}\right)^{(n)} \circ D \circ \tau\right)(a) & \text { if } n \text { is even, }\end{cases}
$$


for all $a \in \mathcal{A}$. Then, for odd $n$,

$$
\begin{aligned}
\widetilde{D}(a c) & =\left(\left(\left.\tau\right|_{\mathcal{I}}\right)^{(n)} \circ D \circ \tau\right)(a c)=\left(\left(\left.\tau\right|_{\mathcal{I}}\right)^{(n)} \circ D\right)(\tau(a) \tau(c)) \\
& =\left(\left.\tau\right|_{\mathcal{I}}\right)^{(n)}(a \cdot D(\tau(c))+D(\tau(a)) \cdot c) \\
& =a \cdot\left(\left(\left.\tau\right|_{I}\right)^{(n)} \circ D(\tau(c))\right)+\left(\left(\left.\tau\right|_{\mathcal{I}}\right)^{(n)} \circ D(\tau(a))\right) \cdot c \\
& =a \cdot \widetilde{D}(c)+\widetilde{D}(a) \cdot c
\end{aligned}
$$

for all $a, c \in \mathcal{A}$. It follows that $\widetilde{D}$ is a derivation from $\mathcal{A}$ into $\mathcal{I}^{(n)}$. By assumption there exists $F \in \mathcal{I}^{(n)}$ such that $\widetilde{D}(a)=\operatorname{ad}_{F}(a)$ for all $a \in \mathcal{A}$. Since $\tau \circ \Phi=I_{\mathcal{B}}$, it follows that $\left.\left.\tau\right|_{\mathcal{I}} \circ \Phi\right|_{\mathcal{J}}=I_{\mathcal{J}}$ and consequently $\left(\left.\tau\right|_{\mathcal{I}}\right)^{(n-1)} \circ\left(\left.\Phi\right|_{\mathcal{J}}\right)^{(n-1)}=I_{\mathcal{J}^{(n-1)}}$. Thus, for each $b \in \mathcal{B}$ and $G \in \mathcal{J}^{(n-1)}$,

$$
\begin{aligned}
\langle D(b), G\rangle & =\left\langle D(\tau \circ \Phi(b)),\left(\left(\left.\tau\right|_{\mathcal{I}}\right)^{(n-1)} \circ\left(\left.\Phi\right|_{\mathcal{J}}\right)^{(n-1)}\right)(G)\right\rangle \\
& =\left\langle\widetilde{D}(\Phi(b)),\left(\left.\Phi\right|_{\mathcal{J}}\right)^{(n-1)}(G)\right\rangle \\
& =\left\langle\Phi(b) \cdot F-F \cdot \Phi(b),\left(\left.\Phi\right|_{\mathcal{J}}\right)^{(n-1)}(G)\right\rangle \\
& =\left\langle\left(\left.\Phi\right|_{\mathcal{J}}\right)^{(n)}(b \cdot F-F \cdot b), G\right\rangle \\
& =\left\langle b \cdot\left(\left.\Phi\right|_{\mathcal{J}}\right)^{(n)}(F)-\left(\left.\Phi\right|_{\mathcal{J}}\right)^{(n)}(F) \cdot b, G\right\rangle .
\end{aligned}
$$

It follows that $D$ is an inner derivation and so $B$ is $n$ - $\mathcal{J}$-weakly amenable. A similar argument holds for even $n$.

As an application of Proposition 2.1 we have the following result.

Corollary 2.2. Let $\mathcal{A}$ be a Banach algebra such that $\mathcal{A}=\mathcal{B} \oplus \mathcal{I}$, the topological sum of $\mathcal{I}$ and $\mathcal{B}$, for some closed two-sided ideal $\mathcal{I}$ and closed subalgebra $\mathcal{B}$. Then $n$-ideal amenability of $\mathcal{A}$ implies that of $\mathcal{B}$.

Proof. First, note that if $\mathcal{J}$ is a closed two-sided ideal in $\mathcal{B}$, then $\mathcal{J} \oplus \mathcal{I}$ is a closed two-sided ideal in $\mathcal{A}$. Let $\tau: \mathcal{A} \rightarrow \mathcal{B}$ be the natural projection and $\Phi: \mathcal{B} \rightarrow \mathcal{A}$ be the natural injection. Then it is clear that $\tau(\mathcal{J} \oplus \mathcal{I}) \subseteq \mathcal{J}$ and $\Phi(\mathcal{J}) \subseteq \mathcal{J} \oplus \mathcal{I}$. So the proof is complete by Proposition 2.1.

Example 2.3. For a Banach algebra $\mathcal{A}$ and a Banach $\mathcal{A}$-module $X$, let $\mathcal{A} \oplus X$ be the module extension Banach algebra which is equipped with the algebra product $(a, x)(b, y)=(a b, a y+x b)(a, b \in \mathcal{A}, x, y \in X)$ and the norm $\|(a, x)\|=\|a\|+\|x\|$; see, for example, [18]. It is clear that $X$ and $\mathcal{A}$ are a closed two-sided ideal and a closed subalgebra of $\mathcal{A} \oplus X$, respectively, and, for each $n \in \mathbb{N}, n$-ideal amenability of $\mathcal{A} \oplus X$ implies that of $\mathcal{A}$ by Corollary 2.2.

Let $\mathcal{A}$ be a Banach algebra. Then $\mathcal{A}^{* *}$, the second dual of $\mathcal{A}$ with first Arens multiplication $\odot$ is a Banach algebra, where $\odot$ is defined by the equations

$$
(F \odot H)(f)=F(H f), \quad(H f)(a)=H(f a), \quad(f a)(b)=f(a b)
$$

for all $F, H \in \mathcal{A}^{* *}, f \in \mathcal{A}^{*}$, and $a, b \in \mathcal{A}$. We recall that a closed $\mathcal{A}$-submodule $X$ of $\mathcal{A}^{*}$ is called left introverted if $\mathcal{A}^{* *} \cdot X \subseteq X$. In this case, $X^{*}$ is a Banach algebra with 
multiplication induced by the left Arens product $\odot$ on $\mathcal{A}^{* *}$. Examples of left introverted subspaces of $\mathcal{A}^{*}$ include $A P(\mathcal{A})$ (almost periodic elements of $\mathcal{A}^{*}$ ), WAP( $\left.\mathcal{A}\right)$ (weakly almost periodic elements of $\left.\mathcal{A}^{*}\right)$, and of course $\mathcal{A}^{*}$. In the case where $\mathcal{A}$ has a bounded right approximate identity, the Cohen-Hewitt factorization theorem shows that $\mathcal{A}^{*} \cdot \mathcal{A}$ is a left introverted subspace of $\mathcal{A}^{*}$.

For every two left introverted subspaces $Y \subseteq X$ of $\mathcal{A}$ it is easy to check that the restriction map $\Theta: X^{*} \rightarrow Y^{*}$ is a continuous homomorphism onto $Y^{*}$ whose kernel is the weak* closed ideal $Y^{\perp}=\left\{F \in X^{*}: F(g)=0\right.$ for all $\left.g \in Y\right\}$ of $X^{*}$; see, for example, [13, Lemma 1.1]. This provides the direct sum decomposition $X^{*}=Y^{*} \oplus Y^{\perp}$. If $Y^{*}$ is isometrically isomorphic to a closed subalgebra of $X^{*}$, we say that the pair $\left(Y^{*}, X^{*}\right)$ is admissible. In this case we have the following result.

Theorem 2.4. Let $\mathcal{A}$ be a Banach algebra and let $X, Y$ be two left introverted subspaces of $\mathcal{A}^{*}$ such that $Y \subseteq X$. If the pair $\left(Y^{*}, X^{*}\right)$ is admissible, then $n$-ideal amenability of $X^{*}$ implies that of $Y^{*}$.

As a consequence we have the following corollary.

Corollary 2.5. Let $\mathcal{A}$ be a dual Banach algebra and let $\mathcal{A}^{* *}$ be $n$-ideally amenable for some $n \in \mathbb{N}$. Then $\mathcal{A}$ is $n$-ideally amenable.

Proof. Let $\mathcal{A}_{*}$ be the predual of $\mathcal{A}$. Then trivially, $\mathcal{A}_{*}$ is an introverted subspace of $\mathcal{A}^{*}$. Thus, $\mathcal{A}^{* *}=\mathcal{A} \oplus\left(\mathcal{A}_{*}\right)^{\perp}$. Since $\left(\mathcal{A}_{*}\right)^{*}=\mathcal{A}$, it follows that the pair $\left(\mathcal{A}, \mathcal{A}^{* *}\right)$ is an admissible pair. Now Proposition 2.4 completes the proof.

Let $\mathcal{A}$ be a Banach algebra with an approximate identity bounded by one. In this case the pair $\left(\left(\mathcal{A}^{*} \cdot \mathcal{A}\right)^{*}, \mathcal{A}^{* *}\right)$ is admissible; see, for example, [10, Corollary 4.2]. So we have the following result.

Corollary 2.6. Let $\mathcal{A}$ be a Banach algebra with an approximate identity bounded by one. Then $n$-ideal amenability of $\mathcal{A}^{* *}$ implies that of $\left(\mathcal{A}^{*} \cdot \mathcal{A}\right)^{*}$.

EXAMPLE 2.7.

(a) Let $G$ be a locally compact group and $\mathcal{A}=L^{1}(G)$. Then $\mathcal{A}^{*}=L^{\infty}(G)$ and $L^{\infty}(G) \cdot L^{1}(G)=L U C(G)$, the algebra of bounded left uniformly continuous functions on $G$. By Corollary 2.6, if $L^{\infty}(G)^{*}$ is $n$-ideal amenable then so is $\operatorname{LUC}(G)^{*}$.

(b) Lau and Loy in their extensive work [13] gave a train of admissible pairs, in particular in the group algebras $L^{\infty}(G), V N(G)$ and $P M_{p}(G)$. Among these admissible pairs are the following.

- $\quad(M(G / N), M(G))$, where $N$ is a compact normal subgroup of $G$.

- $\quad\left(M(G / N), X^{*}\right)$, where $X$ is an introverted subspace of $L^{\infty}(G)$ with $C_{0}(G) \subseteq$ $X \subseteq C(G)$.

- $\quad\left(A P(G)^{*}, W A P(G)^{*}\right)$.

- $\quad\left(W A P(G / N)^{*}, W A P(G)^{*}\right)$, where $N$ is a closed normal subgroup of $G$. 
- $\quad\left(X^{*}, L^{\infty}(G)^{*}\right)$ and $\left(L^{1}(G / N)^{* *}, L^{\infty}(G)^{*}\right)$, in which $G$ is amenable, $N$ is a closed normal subgroup of $G$ and $X$ is a nonzero weak* closed self-adjoint translation invariant subalgebra of $L^{\infty}(G)$. See [8,9] for definitions and details in group algebras.

Proposition 2.8. Let $\mathcal{I}$ and $\mathcal{J}$ be closed two-sided ideals of a Banach algebra $\mathcal{A}$ such that $\mathcal{I} \subseteq \mathcal{J}$. If $\mathcal{I}$ is weakly amenable and $\mathcal{A} / \mathcal{I}$ is $\mathcal{J} / \mathcal{I}$-weakly amenable, then $\mathcal{A}$ is $\mathcal{J}$-weakly amenable.

Proof. Let $\Theta: \mathcal{I} \rightarrow \mathcal{J}$ be the natural embedding map and let $D: \mathcal{A} \rightarrow \mathcal{J}^{*}$ be a continuous derivation. Then $\Theta^{*} \circ D \circ \Theta$ is a continuous derivation from $\mathcal{I}$ into $\mathcal{I}^{*}$. By assumption there exists $f_{0} \in \mathcal{I}^{*}$ such that, for each $\iota \in \mathcal{I}$,

$$
\left(\Theta^{*} \circ D\right)(\iota)=\operatorname{ad}_{f_{0}^{*}}(\iota) .
$$

Let $g_{0}^{*}$ be an extension of $f_{0}^{*}$ on $\mathcal{J}$. If we set $D^{\prime}:=D-\operatorname{ad}_{g_{0}^{*}}$, then $\left.\left(\Theta^{*} \circ D^{\prime}\right)\right|_{\mathcal{I}}=0$. It follows that

$$
\left\langle D^{\prime}\left(\iota^{\prime}\right), b\right\rangle=\left\langle\Theta^{*} \circ D^{\prime}\left(\iota^{\prime}\right), b \iota\right\rangle+\left\langle\Theta^{*} \circ D(\iota), \iota^{\prime} b\right\rangle
$$

for all $\iota, \iota^{\prime} \in \mathcal{I}$ and $b \in \mathcal{J}$. This shows that $\left.D^{\prime}\right|_{I^{2}}=0$. On the other hand, by [4, Theorem 2.8.63(i)], $\overline{\mathcal{I}^{2}}=\mathcal{I}$, and so $\left.D^{\prime}\right|_{\mathcal{I}}=0$. It follows that for each $a \in \mathcal{A}$ and $\iota \in \mathcal{I}$,

$$
a \cdot D^{\prime}(\iota)=D^{\prime}(a \iota)=0
$$

and so $D^{\prime}(a) \cdot \iota=0$. Consequently, for each $\iota, \iota^{\prime} \in \mathcal{I}$,

$$
\left\langle D^{\prime} a, \iota^{\prime}\right\rangle=\left\langle D^{\prime} a \cdot \iota, \iota^{\prime}\right\rangle=0
$$

that is, $\left.D^{\prime} a\right|_{I^{2}}=0$ and hence $\left.D^{\prime} a\right|_{I}=0$. Thus $D^{\prime}(\mathcal{A}) \subseteq \mathcal{I}^{\perp}$. Define the map $\widetilde{D}: \mathcal{A} / \mathcal{I} \rightarrow$ $\mathcal{I}^{\perp}$ by

$$
\widetilde{D}(a+\mathcal{I})=D^{\prime}(a) \quad(a \in \mathcal{A}) .
$$

It is easy to check that $\widetilde{D}$ is a continuous derivation. By hypothesis, there exists an element $g_{1} \in \mathcal{I}^{\perp} \subseteq \mathcal{J}^{*}$ such that

$$
D^{\prime} a=a \cdot g_{1}-g_{1} \cdot a \quad(a \in \mathcal{A}) .
$$

Therefore $D(a)=\operatorname{ad}_{g_{0}+g_{1}}$ for all $a \in \mathcal{A}$; that is, $\mathcal{A}$ is $\mathcal{J}$-weakly amenable.

\section{Approximate $\boldsymbol{n}$-ideal amenability of abstract Segal algebras}

Let $\mathcal{A}$ be a Banach algebra with the norm $\|\cdot\|_{\mathcal{A}}$. Then a Banach algebra $\mathcal{B}$ with the norm $\|\cdot\|_{\mathcal{B}}$ is an abstract Segal algebra in $\mathcal{A}$ if:

(1) $\mathcal{B}$ is a dense left ideal in $\mathcal{A}$;

(2) there exists $M>0$ such that $\|b\|_{\mathcal{A}} \leq M\|b\|_{\mathcal{B}}$ for each $b \in \mathcal{B}$;

(3) there exists $C>0$ such that $\|a b\|_{\mathcal{B}} \leq C\|a\|_{\mathcal{A}}\|b\|_{\mathcal{B}}$ for all $a, b \in \mathcal{B}$. 
We further say that $\mathcal{B}$ is symmetric if it is also a two-sided dense ideal in $\mathcal{A}$ and $\|b a\|_{\mathcal{B}} \leq C\|a\|_{\mathcal{A}}\|b\|_{\mathcal{B}}$ for all $a, b \in \mathcal{B}$.

ReMARK 3.1. It was shown in [14] that if $\mathcal{B}$ is an abstract Segal algebra in $\mathcal{A}$, then the mapping $\mathcal{J} \mapsto \overline{\mathcal{J}}^{\mathcal{A}}$ is a bijection from the set of all closed two-sided ideals in $\mathcal{B}$ onto the set of all closed two-sided ideals in $\mathcal{A}$ and the inverse mapping is $\mathcal{I} \mapsto \mathcal{I} \cap \mathcal{B}$, where for a set $\mathcal{J} \subset \mathcal{B}$ the notation $\overline{\mathcal{J}}^{\mathcal{A}}$ stands for the closure of $\mathcal{J}$ in $\mathcal{A}$; see also [1].

We commence this section with the following result.

THeOREM 3.2. Let $\mathcal{B}$ be an abstract Segal algebra in a Banach algebra $\mathcal{A}$ with a central approximate identity which is bounded in $\|\cdot\|_{\mathcal{A}}$ and let $\mathcal{J}$ be a closed two-sided ideal in $\mathcal{B}$. Set $\mathcal{I}=\overline{\mathcal{J}}^{\mathcal{A}}$. Then for each $n \in \mathbb{N}$, the following statements hold.

(a) If $\mathcal{A}$ is $n$ - $\mathcal{I}$-weakly amenable, then $\mathcal{B}$ is approximately $n$ - $\mathcal{J}$-weakly amenable.

(b) If $\mathcal{A}$ is $n$-ideally amenable, then $\mathcal{B}$ is approximately $n$-ideally amenable.

Proof. (a) Let $n \in \mathbb{N}$ and $D: \mathcal{B} \rightarrow \mathcal{J}^{(n)}$ be a continuous derivation. Then we denote by $X_{n}$ the closed linear span of the set $\left\{a \cdot \mathcal{J}^{(n)} \cdot b: a, b \in \mathcal{B}\right\}$. Suppose that $\left(e_{\alpha}\right)$ is a central approximate identity and bounded by $\|\cdot\|_{\mathcal{A}}$ for $\mathcal{B}$. Since $\left(e_{\alpha}\right)$ is central,

$$
D(\mathcal{B}) \subseteq X_{n}
$$

Note that $\left(e_{\alpha}\right)$ is a multiplier-bounded, central approximate identity for $X_{n}$. In particular,

$$
\lim _{\alpha} e_{\alpha}^{2} \cdot D(b)=D(b) \quad(b \in \mathcal{B}) .
$$

For each $\alpha$, define the map $\tau_{\alpha}: \mathcal{I} \rightarrow \mathcal{J}$ by

$$
\tau_{\alpha}(a)=a e_{\alpha} \quad(a \in \mathcal{I})
$$

and let $\theta: \mathcal{J} \rightarrow \mathcal{I}$ denote the inclusion map. Trivially, both $\tau_{\alpha}$ and $\theta$ are linear and continuous left $\mathcal{A}$-bimodule morphisms and also continuous $\mathcal{B}$-bimodule morphisms. It is clear that $\tau_{\alpha} \theta(b)=b e_{\alpha}=e_{\alpha} b$ for all $b \in \mathcal{J}$, so by induction, for each $n \in \mathbb{N}$,

$$
\left(\tau_{\alpha} \theta\right)^{(n)}(F)=F \cdot e_{\alpha}=e_{\alpha} \cdot F \quad\left(F \in \mathcal{J}^{(n)}\right) .
$$

Define the continuous linear map $D_{\alpha}: \mathcal{A} \rightarrow \mathcal{I}^{(n)}$ by

$$
D_{\alpha}(a)= \begin{cases}\theta^{(n)}\left[D\left(a e_{\alpha}\right)-a \cdot D\left(e_{\alpha}\right)\right] & \text { if } n \text { is even, } \\ \tau_{\alpha}^{(n)}\left[D\left(e_{\alpha} a\right)-D\left(e_{\alpha}\right) \cdot a\right] & \text { if } n \text { is odd }\end{cases}
$$

for all $a \in \mathcal{A}$. Then, for each $b \in \mathcal{B}$,

$$
D_{\alpha}(b)= \begin{cases}\theta^{(n)}\left(D(b) \cdot e_{\alpha}\right) & \text { if } n \text { is even } \\ \tau_{\alpha}^{(n)}\left(e_{\alpha} \cdot D(b)\right) & \text { if } n \text { is odd }\end{cases}
$$


Since $e_{\alpha}$ is central, it follows that $D_{\alpha}(b c)=b \cdot D(c)+D(b) \cdot c$ for all $b, c \in \mathcal{B}$. Since $\mathcal{B}$ is dense in $\mathcal{A}$, it follows that $D_{\alpha}$ is a derivation from $\mathcal{A}$ into $\mathcal{I}^{(n)}$. By assumption there exists $F_{\alpha} \in \mathcal{I}^{(n)}$ such that

$$
D_{\alpha}(a)=a \cdot F_{\alpha}-F_{\alpha} \cdot a \quad(a \in \mathcal{A}) .
$$

In particular, by (3.2), for even $n$,

$$
\begin{aligned}
D(b) \cdot e_{\alpha}^{2} & =\left(\tau_{\alpha} \theta\right)^{(n)}\left(D(b) \cdot e_{\alpha}\right) \\
& =\tau_{\alpha}^{(n)} D_{\alpha}(b) \\
& =b \cdot \tau_{\alpha}^{(n)}\left(F_{\alpha}\right)-\tau_{\alpha}^{(n)}\left(F_{\alpha}\right) \cdot b,
\end{aligned}
$$

and for odd $n$,

$$
\begin{aligned}
e_{\alpha}^{2} \cdot D(b) & =\left(\tau_{\alpha} \theta\right)^{(n)}\left(e_{\alpha} \cdot D(b)\right) \\
& =\theta^{(n)} D_{\alpha}(b) \\
& =b \cdot \theta^{(n)}\left(F_{\alpha}\right)-\theta^{(n)}\left(F_{\alpha}\right) \cdot b,
\end{aligned}
$$

for all $b \in \mathcal{B}$. Set $G_{\alpha}=\tau_{\alpha}^{(n)}\left(F_{\alpha}\right)$ if $n$ is even and set $G_{\alpha}=\theta^{(n)}\left(F_{\alpha}\right)$ if $n$ is odd. Trivially, $G_{\alpha} \in \mathcal{I}^{(n)}$ for all $\alpha$ and we have, by (3.1),

$$
D(b)=\lim _{\alpha} e_{\alpha}^{2} \cdot D(b)=\lim _{\alpha} b \cdot G_{\alpha}-G_{\alpha} \cdot b \quad(b \in \mathcal{B})
$$

that is, $D$ is approximately inner.

(b) This follows immediately from part (a) and Remark 3.1.

Let $G$ be a locally compact group. A linear subspace $S(G)$ of the convolution group algebra $L^{1}(G)$ is said to be a Segal algebra on $G$ if it satisfies the following conditions:

(1) $S(G)$ is dense in $L^{1}(G)$;

(2) $S(G)$ is a Banach space under some norm $\|\cdot\|_{S}$ and there exists $M>0$ such that

$$
\|f\|_{1} \leq\|f\|_{S} \quad \text { for all } f \in S(G)
$$

(3) $S(G)$ is left transition invariant and the map $x \mapsto \delta_{x} * f$ of $G$ into $S(G)$ is continuous;

(4) $\left\|\delta_{x} * f\right\|_{S}=\|f\|_{S}$ for all $f \in S(G)$ and $x \in G$.

A Segal algebra $S(G)$ is symmetric if it is right translation invariant, and for each $f \in S(G),\left\|f * \delta_{x}\right\|_{S}=\|f\|_{S}$ for all $x \in G$, and the map $x \mapsto f * \delta_{x}$ from $G$ into $S(G)$ is continuous.

It is well known that a Segal algebra on $G$ is an abstract Segal algebra; see [3, p. 492].

We call $G$ a SIN group if there is a basis for the neighborhood of the identity of $G$ consisting of compact sets $U$ such that $x U x^{-1}=U$ for all $x \in G$. 
Corollary 3.3. Let $S(G)$ be a Segal algebra on a locally compact SIN group $G$ and let $\mathcal{J}$ be a closed two-sided ideal in $S(G)$ such that $\mathcal{I}=\overline{\mathcal{J}}^{L^{1}(G)}$. Then for each $n \in \mathbb{N}$ the following statements hold.

(a) If $L^{1}(G)$ is $n$-I-weakly amenable, then $S(G)$ is approximately $n$ - $\mathcal{J}$-weakly amenable.

(b) If $L^{1}(G)$ is $n$-ideally amenable, then $S(G)$ is approximately $n$-ideally amenable.

Proof. Since $G$ is a SIN group, then by a result of [12], $S(G)$ has a central approximate identity which is bounded in $\|\cdot\|_{1}$ and the result is obvious from Theorem 3.2.

Corollary 3.4. Let $S(G)$ be a Segal algebra on a locally compact, amenable SIN group $G$. Then $S(G)$ is approximately permanently ideally amenable.

Proof. Since $G$ is amenable, Johnson's theorem implies that $L^{1}(G)$ is amenable, in particular it is permanently ideally amenable. Now, Corollary 3.3 completes the proof.

We know that a commutative Banach algebra is weakly amenable if and only if every derivation from $\mathcal{A}$ into a commutative Banach $\mathcal{A}$-module is zero; see, for example, [2, Theorem 1.5]. Thus we have the following result.

Theorem 3.5. Let $\mathcal{A}$ be a commutative weakly amenable Banach algebra. Then any abstract Segal algebra $\mathcal{B}$ in $\mathcal{A}$ having an approximate identity, which is bounded in $\|\cdot\|_{\mathcal{A}}$, is permanently ideally amenable.

Corollary 3.6. Every Segal algebra on an abelian locally compact group is permanently ideally amenable.

Theorem 3.7. Let $\mathcal{B}$ be a symmetric abstract Segal algebra in an amenable Banach algebra $\mathcal{A}$. Then the following statements are equivalent.

(a) $\mathcal{B}$ is approximately ideally amenable.

(b) $\mathcal{B}$ has an approximate identity.

Proof. $(a) \Rightarrow(b)$. Since $\mathcal{B}$ is approximately ideally amenable, it is obviously approximately weakly amenable, so by [17, Theorem 2.7], it has an approximate identity.

$(b) \Rightarrow(a)$. This follows by the same argument used in the proof of [7, Theorem 3.1].

Recall that the symmetric Segal algebras on a locally compact group include all Segal algebras on locally compact abelian groups. Also, every such symmetric Segal algebra is a two-sided ideal in $L^{1}(G)$ and has an approximate identity which is bounded in $\|\cdot\|_{1}$; see [16] for details. We have the following result for symmetric Segal algebras on $G$.

Corollary 3.8. Let $G$ be an amenable locally compact group and let $S(G)$ be a symmetric Segal algebra on $G$. Then $S(G)$ is approximately ideally amenable. 
THEOREM 3.9. Let $G$ be a compact group and $2 \leq p<\infty$. Then the convolution algebra $L^{p}(G)$ is ideally amenable if and only if $G$ is abelian or finite.

Proof. First suppose that $L^{p}(G)$ is ideally amenable. Then obviously it is weakly amenable. Now by [17, Proposition 3.4], $G$ is abelian or finite.

Conversely, note that in this case $L^{p}(G)$ is a symmetric Segal algebra on $G$. Suppose that $G$ is abelian or finite. If $G$ is abelian, then by Corollary 3.6, $L^{p}(G)$ is ideally amenable. If $G$ is finite, then $l^{p}(G)=l^{1}(G)$ is amenable and in particular is ideally amenable.

\section{Acknowledgement}

The authors thank Islamic Azad University, Khorasgan branch, for its financial support.

\section{References}

[1] M. Alaghmandan, R. Nasr-Isfahani and M. Nemati, 'Character amenability and contractibility of abstract Segal algebras', Bull. Aust. Math. Soc. 82 (2010), 274-281.

[2] W. G. Bade, P. C. Curtis and H. G. Dales, 'Amenability and weak amenability for Beurling and Lipschitz algebras', Proc. Lond. Math. Soc. 55 (1987), 359-377.

[3] H. G. Dales, Banach Algebras and Automatic Continuity (Clarendon Press, Oxford, 2000).

[4] H. G. Dales, F. Ghahramani and N. Grønbæk, 'Derivations into iterated duals of Banach algebras', Studia Math. 128 (1998), 19-53.

[5] M. Eshaghi Gordji and R. Memarbashi, 'Derivations into $n$th duals of ideals of Banach algebras', Bull. Iran Math. Soc. 34 (2008), 59-71.

[6] M. Eshaghi Gordji and T. Yazdanpanah, 'Derivations into duals of ideals of Banach algebras', Proc. Indian Acad. Sci. 114 (2004), 399-408.

[7] F. Ghahramani and A. T.-M. Lau, 'Approximate weak amenability, derivations and Arens regularity of Segal algebras', Studia Math. 169 (2005), 189-205.

[8] E. Hewitt and K. A. Ross, Abstract Harmonic Analysis, Vol. I, Grundlehren der Mathematischen Wissenschaften, 152 (Springer, Berlin, 1970).

[9] E. Hewitt and K. A. Ross, Abstract Harmonic Analysis, 2nd edn, Vol. II, Grundlehren der Mathematischen Wissenschaften, 115 (Springer, Berlin, 1979).

[10] A. Jabbari, M. S. Moslehian and H. R. E. Vishki, 'Constructions preserving $n$-weak amenability of Banach algebras', Math. Bohem. 134 (2009), 349-357.

[11] B. E. Johnson, 'Derivations from $L^{1}(G)$ into $L^{1}(G)$ and $L^{\infty}(G)$ ', in: Proc. Internat. Conf. on Harmonic Analysis, Luxembourg 1987, Lecture Notes in Mathematics, 1359 (Springer, BerlinNew York, 1988), pp. 191-198.

[12] E. Kotzmann and H. Rindler, 'Segal algebras on non-abelian groups', Trans. Amer. Math. Soc. 237 (1978), 271-281.

[13] A. T.-M. Lau and R. J. Loy, 'Weak amenability of Banach algebras on locally compact groups', J. Funct. Anal. 145 (1997), 175-204.

[14] M. Leinert, 'A contribution to Segal algebras', Manuscripta Math. 10 (1973), 297-306.

[15] O. T. Mewomo, 'On approximate ideal amenability in Banach algebras', Mathematică LVI (2010), 199-208.

[16] H. Reiter, L1-Algebras and Segal Algebras, Lecture Notes in Mathematics, 231 (Springer, Berlin, 1971).

[17] H. Samea, 'Approximate weak amenability of abstract Segal algebras', Math. Scand 106 (2010), 243-249.

[18] Y. Zhang, 'Weak amenability of module extensions of Banach algebras', Trans. Amer. Math. Soc. 354 (2010), 4131-4151. 
ZEINAB KAMALI, Department of Mathematical Sciences, Islamic Azad University, Khorasgan branch, Isfahan, Iran

e-mail: zkamali@ualberta.ca

MEHDI NEMATI, Department of Mathematics, Faculty of Science,

University of Kashan, Kashan 87317-51167, Iran

e-mail: m.nemati@math.iut.ac.ir 